\title{
Is There a Role for Sonde Enteroscopy in Patients with Ob- scure Gastrointestinal Bleeding? A Comparison with Capsule Endoscopy
}

\author{
Samuel N Giordano ${ }^{\mathrm{a}, \mathrm{b}}$, Jeff Dilisi ${ }^{\mathrm{a}}$, Kuldip Banwait ${ }^{\mathrm{a}}$, Daniel Wild ${ }^{\mathrm{a}}$, \\ Anthony Infantolino ${ }^{a}$, Lenore Miranda ${ }^{\mathrm{a}}$, Mitchell Conn ${ }^{\mathrm{a}}$
}

\begin{abstract}
Background: In the 1980's and 1990's combined Push and Sonde Enteroscopy was the primary endoscopic tool used to evaluate the small intestine in patients with obscure gastrointestinal bleeding (OGIB). It was available in only a few centers due to the technical difficulties associated with its use. The introduction of wireless capsule endoscopy in 2001 revolutionalized small bowel endoscopic imaging making Sonde enteroscopy a rarely used procedure despite the lack of studies comparing the efficacy of the two modalities. The aim of this study was to restrospectively compare the findings of Sonde enteroscopy with capsule endoscopy in patients with OGIB.
\end{abstract}

Methods: Design: One hundred patients who underwent Sonde enteroscopy and 101 patients who underwent capsule endoscopy were retrospectively studied. Setting: All patients had their procedures completed by physicians within the same gastroenterology practice. Patients: All patients who underwent either Sonde enteroscopy or capsule endoscopy were enrolled. Interventions: None. Main outcome measurements: Outcome was defined as the number of patients in which a distinct bleeding site could be identified.

Results: A total of 100 patients underwent Push and Sonde enteroscopy and a potential bleeding site was identified in 55 (55\%) patients. A total of 101 patients underwent capsule endoscopy and a potential bleeding site was identified in $60(59 \%)$ patients. A onetailed $\mathrm{P}$ value showed no statistically significant difference in the diagnostic yield between the procedures.

Manuscript accepted for publication April 16, 2009.

${ }^{a}$ Division of Gastroenterology and Hepatology, Thomas Jefferson University Hospital, 132 S. 10th Street, 480 Main Building, Philadelphia, PA 19107, USA

${ }^{b}$ Corresponding author: 1805 Roberts Way, Voorhees NJ 08043, USA.

Email: Samuel giordano@hotmail.com

doi:10.4021/gr2009.04.1284
Conclusions: A total of 100 patients underwent Push and Sonde enteroscopy and a potential bleeding site was identified in $55(55 \%)$ patients. A total of 101 patients underwent capsule endoscopy and a potential bleeding site was identified in $60(59 \%)$ patients. A onetailed $\mathrm{P}$ value showed no statistically significant difference in the diagnostic yield between the procedures.

Keywords: Sonde; capsule endoscopy; Obscure gastrointestinal bleeding; Enteroscopy

\section{Introduction}

Obscure gastrointestinal bleeding (OGIB) is defined as bleeding in which the source remains unknown following initial evaluation with upper endoscopy and colonoscopy [1]. OGIB accounts for $3 \%$ to $5 \%$ of cases of gastrointestinal bleeding $[2,3]$. The majority of these patients are ultimately found to be bleeding from the small intestine.

Complete endoscopic examination of the small intestine has been difficult due to its length and tortuosity. Push enteroscopy utilizing pediatric and adult colonoscopes and specialized small bowel enteroscopes have been used to examine the proximal small intestine. Diagnostic yield with push enteroscopy has ranged from $26 \%$ to $75 \%$ in several studies [4-9]. In the late 1980's, Sonde enteroscopy was introduced and used to visualize the small bowel beyond the reach of the push enteroscope. Combined Push/Sonde enteroscopy resulted in a diagnostic yield of $40 \%-50 \%$ [10-12]. Despite these favorable results, Sonde enteroscopy was not universally accepted and only performed at a few institutions. The procedure was perceived as draconian associated with patient discomfort, labor intensive requiring hours of physician time, purely diagnostic lacking therapeutic capabilities and variable in its ability to visualize the most distal small intestine.

In 2001 when small bowel capsule endoscopy was introduced, it rapidly supplanted Sonde enteroscopy and became the endoscopic procedure of choice for visualization of the small bowel. Studies confirmed its superiority over push enteroscopy. The diagnostic yield of capsule endoscopy has 
been reported to range from $55 \%$ to $68 \%$, compared to push enteroscopy, which in randomized studies, had a diagnostic yield of 28\%-32\% [13-15]. In 2003, Dr. Jerry Waye proclaimed that Sonde Enteroscopy can be laid to rest [16] despite the lack of any comparison between the two techniques appearing in the literature.

In 2004, Double Balloon Enteroscopy was introduced offering a therapeutic modality to small bowel pathology. Early reports have demonstrated its diagnostic and therapeutic benefits $[17,18]$. It would seem appropriate then, with the development of both capsule endoscopy and double balloon enteroscopy that Sonde Enteroscopy can be laid to rest along side the Gavin Balloon and the gallstone lithotripter. However, without a comparison study we may overlook any benefits or advantages Sonde enteroscopy may have had. One could argue that Sonde enteroscopy allowed direct physician involvement and afforded some level of control of the endoscope during visualization and withdrawal when compared with the capsule. Sonde enteroscopy had air insufflation and water irrigation capabilities. In light of this, the aim of our study is to compare our experience with Sonde enteroscopy and capsule endoscopy in patients with obscure intestinal bleeding at our institution.

\section{Materials and Methods}

Prior to the development of capsule endoscopy, the gold standard at our institution was to perform Sonde enteroscopy to evaluate patients with OGIB. Our technique of performing Sonde enteroscopy has been previously reported [19]. All patients we evaluated undergoing Sonde enteroscopy also had a push enteroscopy performed on the same day. Extent of insertion of the Sonde Enteroscope was estimated by imaging with a plain abdominal X-ray prior to removal of the instrument. Potential bleeding sites were defined as abnormalities known to cause bleeding such as vascular ectasias, ulcers, erosions and tumors. Lymphangiectasias, lipomas, and diverticulum were not considered as potential causes of bleeding. One endoscopist performed all Push/Sonde enteroscopies (PSE) during the time period of June 1993 through February 2001. A total of 100 consecutive patients who underwent PSE were included in this study. Since December 2001, we have been performing capsule endoscopy (Given SB PillCam) for the evaluation of patients with obscure gastrointestinal bleeding. The technique for performing and reading capsule endoscopy studies has been previously reported [20, 21]. The majority of patients referred to our hospital for capsule endoscopy have already undergone extensive endoscopic evaluation prior to our evaluation. The first 101 patients undergoing capsule endoscopy for obscure GI bleeding from December, 2001 to January, 2003 were included in our study. Relevant data and history on these patients was collected prior to the procedure, including the age and gender of the patient, clinical presentation, previous endoscopic evaluation(s), blood transfusion requirements, and procedure findings.

We performed a retrospective analysis between patients who underwent Push/Sonde enteroscopy compared with those undergoing capsule endoscopy. A two-tail t-test analysis was performed comparing PSE and Sonde alone to capsule endoscopy regarding these variables and each procedure's diagnostic yield. The study was reviewed by the IRB committee at our institution and was approved.

Table 1. Overview of demographics and presenting complaints of PSE and M2A groups

\begin{tabular}{lll}
\hline & PSE (N=100) & M2A Capsule (N= 101) \\
\hline Men-no. (\%) & $45(45)$ & $45(44)$ \\
Women-no. (\%) & $55(55)$ & $55(56)$ \\
Age-yr & $67.3(30-87)$ & $65.2(13-87)$ \\
Chief presenting complaint & & \\
$\quad$ Melena-no.(\%) & $46(46)$ & $67(67)$ \\
Occult Bleeding-no. (\%) & $39(39)$ & $6(6)$ \\
Hematochezia-no. (\%) & $15(15)$ & $62(62)$ \\
Patients requiring previous transfusions-no. (\%) & $73(73)$ & \\
\hline
\end{tabular}

M2A, mouth-to-anus. 


\section{Results}

A total of 100 patients who underwent PSE during the time period of June 1993 through February, 2001 were included in the retrospective chart review. The mean age of these patients was 67.3 years (range $30-87$ ). Fifty-five percent of the patients were women and $45 \%$ were men. Among these patients, $46 \%$ presented with melena, $39 \%$ presented with occult bleeding, and 15\% with hematochezia (Table 1). Seventy-three percent of these patients required prior blood transfusions and this group averaged 7.1 procedures before Sonde while the $27 \%$ not requiring transfusions averaged 4.9 procedures prior to Sonde.

It was estimated that Sonde enteroscopy visualized into the ileum in $50 \%$ of the patients, with the distal ileum being visualized in 7\%. Combined PSE identified 60 potential bleeding sites in 55 patients with an overall yield of $55 \%$. Findings included: vascular ectasias in 38 patients $(69.09 \%)$; active bleeding in 2 patients (3.64\%); ulcers/erosions in 13 patients $(23.63 \%)$; and tumors in 7 patients $(12.72 \%)$; (Table 2).

Sonde enteroscopy alone identified a potential bleeding site in 43 patients, resulting in an overall yield of $43 \%$. Among the 43 patients who had diagnoses identified by Sonde enteroscopy alone, 26 patients had vascular ectasias (60.46\%); 2 had active bleeding (4.65\%); 9 had ulcers $(20.90 \%)$; 4 had tumors (9.34\%); and $2 \mathrm{had}$ erosions $(4.65 \%)$. The location of these findings can be found in Table 3 .

A total of 101 patients undergoing capsule endoscopy for the evaluation of OGIB from December, 2001 through January, 2003 were examined. The mean age of presenta- tion for patients undergoing this procedure was 65.2 years (range 13-89), of which, 56\% were women and 44\% were men. Patients presented with melena, occult bleeding, and hematochezia were $27 \%, 67 \%$, and $6 \%$ respectively. Sixtytwo percent of these patients required blood transfusions prior to capsule endoscopy. The majority of patients for capsule endoscopy were referred from outside institutions as a result the number of procedures prior to capsule endoscopy was not available.

The capsule successfully visualized the entire small intestine and passed into the colon in $88.7 \%$ of the patients. All capsule passed spontaneously, there were no patients with retained capsules. A potential bleeding site was identified in 60 patients $(59 \%)$. These findings included vascular ectasias in 34 patients (56.67\%); active bleeding in 9 patients $(15 \%)$; suspected Crohn's disease in 6 patients $(10 \%)$; ulcers in 7 patients (11.67\%); tumors in 3 patients $(5 \%)$; and an hemagioma in 1 patient (1.67\%).

Overall, there was no statistical difference in diagnostic yield between PSE and capsule endoscopy ( $\mathrm{P}>0.05$ ). Capsule endoscopy visualized the small intestine more completely than push/sonde reaching the colon in $88.7 \%$ of patients. As expected, PSE never reached the colon. When the specific causes of bleeding were compared in the PSE group versus the capsule group, the capsule identified active bleeding more frequently than PSE, $\mathrm{P}<0.05$. No diagnoses of Crohn's disease or hemangioma were made in the PSE group. More patients in the PSE group presented with active bleeding, i.e. hematochezia $15 \%$ versus $6 \%$ in the capsule group.

Table 2. Comparison of findings between PSE and M2A

\begin{tabular}{llll}
\hline & $\begin{array}{l}\text { Sonde Enteroscopy } \\
(\mathbf{n}=\mathbf{1 0 0})\end{array}$ & $\begin{array}{l}\text { Push/Sonde Enteroscopy } \\
(\mathbf{n}=\mathbf{1 0 0})\end{array}$ & $\begin{array}{l}\text { M2A Capsule } \\
(\mathbf{n}=\mathbf{1 0 1})\end{array}$ \\
\hline $\begin{array}{l}\text { Patients identified with potential bleeding } \\
\text { site }\end{array}$ & $43 \%(\mathrm{n}=43)$ & $55 \%(\mathrm{n}=55)$ & $59 \%(\mathrm{n}=60)$ \\
Vascular Ectasias & $60.46 \%(\mathrm{n}=26)$ & $69.09 \%(\mathrm{n}=38)$ & $56.67 \%(\mathrm{n}=34)$ \\
$\begin{array}{l}\text { Active Bleeding } \\
\text { Ulcer/Erosion }\end{array}$ & $\begin{array}{l}4.65 \%(\mathrm{n}=2) \\
\text { Tumor }\end{array}$ & $3.64 \%(\mathrm{n}=2)$ & $15 \%(\mathrm{n}=9)$ \\
$\begin{array}{l}\text { Hemangioma } \\
\text { Crohn's Disease }\end{array}$ & $0 \%(\mathrm{n}=0)$ & $23.63 \%(\mathrm{n}=13)$ & $11.67 \%(\mathrm{n}=7)$ \\
\hline
\end{tabular}

M2A, mouth-to-anus. 
Table 3. Location of Sonde enteroscopy findings

\begin{tabular}{|c|c|c|c|c|}
\hline Finding & Total findings & Duodenum & Jejunum & Ileum \\
\hline Vascular ectasias & 26 & $7.69 \%(n=2)$ & $73.07 \%(\mathrm{n}=19)$ & $19.23 \%(n=5)$ \\
\hline Ulcers & 9 & $0 \%(\mathrm{n}=0)$ & $88.89 \%(\mathrm{n}=8)$ & $12.11 \%(n=1)$ \\
\hline Tumors & 4 & $0 \%(\mathrm{n}=0)$ & $75 \%(n=3)$ & $25 \%(\mathrm{n}=1)$ \\
\hline Erosions & 2 & $0 \%(n=0)$ & $100 \%(n=2)$ & $0 \%(n=0)$ \\
\hline Active Bleeding & 2 & $0 \%(\mathrm{n}=0)$ & $100 \%(n=2)$ & $0 \%(\mathrm{n}=0)$ \\
\hline
\end{tabular}

\section{Discussion}

The aim of our study was to compare capsule endoscopy to PSE for the evaluation of OGIB. This comparison is important as capsule endoscopy has replaced combined PSE as the gold standard for evaluating patients with OGIB; however, a direct comparison has not been published in the literature. We found that the diagnostic yield of the PSE and capsule endoscopy was not significantly different in our study despite the fact that the capsule was found to examine a greater extent of the small intestine. The lack of difference in these findings can be explained in several ways. At the time of this study, capsule endoscopy was still a relatively new technology and therefore an initial learning period may have been required prior to optimizing its yield. In addition, more than one gastroenterologist contributed readings of capsule studies compared to PSE studies, which were all performed by only one gastroenterologist at our institution. Another explanation is that the distribution of bleeding sites in the small intestine may have a proximal predilection, with most of the OGIB sources being in the duodenum and jejunum, which are often within the reach of push/Sonde enteroscopy [2, 22]. Direct physician involvement in performing both push and Sonde enteroscopy may enhance its diagnostic yield, when compared with the observational nature of the capsule. These explanations could certainly explain why there was no overall difference in the diagnostic yield of PSE when compared to capsule for the diagnosis of OGIB.

We acknowledge certain weakness in our study. First this is a retrospective review of two different populations of patients. These two groups were different in the manner in which they presented for further endoscopic evaluation of obscure gastrointestinal bleeding. The patients in the PSE group presented more often with overt bleeding, documented as melena and hematochezia than the patients in the capsule group, who presented most often with occult bleeding.
There has been a clear change in referral pattern since the introduction of capsule endoscopy. Sonde enteroscopy was reserved for the clinically more significant bleeding whereas clinically less significant bleeders are now being referred for capsule studies. If the PSE group is a higher risk group, then the diagnostic yield could approach that seen in the capsule group, not because it is as sensitive, but because the patients are more likely to have abnormalities.

A greater number of patients with active bleeding were identified by capsule endoscopy compared to PSE (P $<0.05$ ), despite the fact that more patients presented with active bleeding in the PSE group. One explanation for this discrepancy might be that capsule studies are sedation free. Consequently, the capsule captures the appearance of the bowel mucosa in its natural state, rather than under the drug effects of conscious sedation, which can lead to attenuation of bleeding secondary to the vasoconstrictive effects.

Our findings suggest that capsule endoscopy is at least as efficacious as PSE in evaluating patients with OGIB. In addition, capsule endoscopy was shown to visualize more of the small intestine. Capsule endoscopy may also be better at diagnosing Crohn's Disease than PSE, as no cases were observed in the PSE group compared to $10 \%$ in the capsule group. This probably reflects the capsule's ability to more consistently visualize the distal ileum where Crohn's Disease is most likely to occur. Recent studies support this evidence by demonstrating the diagnostic yield of capsule endoscopy in the diagnosis of Crohn's disease compared to other modalities [23-25]. Sonde enteroscopy is no longer used at our institution given the capsule's safety, avoidance of sedation, ease of use and overall effectiveness. With the introduction of Double Balloon Enteroscopy and its' proven diagnostic and therapeutic abilities, we now utilize capsule endoscopy as an initial diagnostic tool for OGIB followed by Push or Double Balloon enteroscopy if findings on capsule study warrant its use. We can now comfortably lay to rest 
Sonde enteroscopy. May it rest in peace.

\section{Acknowledgements}

The authors declare no commercial associations or conflict of interests related to this article.

\section{References}

1. Zuckerman GR, Prakash C, Askin MP, Lewis BS. AGA technical review on the evaluation and management of occult and obscure gastrointestinal bleeding. Gastroenterology 2000;118:201-221.

2. Netterville RE, Hardy JD, Martin RS, Jr. Small bowel hemorrhage. Ann Surg 1968;167:949-957.

3. Lewis BS. Small intestinal bleeding. Gastroenterol Clin North Am 2000;29:67-95, vi.

4. Taylor AC, Chen RY, Desmond PV. Use of an overtube for enteroscopy--does it increase depth of insertion? A prospective study of enteroscopy with and without an overtube. Endoscopy 2001;33:227-230.

5. Barkin JS, Lewis BS, Reiner DK, Waye JD, Goldberg RI, Phillips RS. Diagnostic and therapeutic jejunoscopy with a new, longer enteroscope. Gastrointest Endosc 1992;38:55-58.

6. Yang R, Laine L. Mucosal stripping: a complication of push enteroscopy. Gastrointest Endosc 1995;41:156158.

7. Linder J, Cheruvattath R, Truss C, Wilcox CM. Diagnostic yield and clinical implications of push enteroscopy: results from a nonspecialized center. J Clin Gastroenterol 2002;35:383-386.

8. Parry SD, Welfare MR, Cobden I, Barton JR. Push enteroscopy in a UK district general hospital: experience of 51 cases over 2 years. Eur J Gastroenterol Hepatol 2002;14:305-309.

9. Taylor AC, Buttigieg RJ, McDonald IG, Desmond PV. Prospective assessment of the diagnostic and therapeutic impact of small-bowel push enteroscopy. Endoscopy 2003;35:951-956.

10. Foutch PG, Sawyer R, Sanowski RA. Push-enteroscopy for diagnosis of patients with gastrointestinal bleeding of obscure origin. Gastrointest Endosc 1990;36:337341.

11. Lewis BS, Wenger JS, Waye JD. Small bowel enteroscopy and intraoperative enteroscopy for obscure gastrointestinal bleeding. Am J Gastroenterol 1991;86:171-174.

12. Zaman A, Katon RM. Push enteroscopy for obscure gas- trointestinal bleeding yields a high incidence of proximal lesions within reach of a standard endoscope. Gastrointest Endosc 1998;47:372-376.

13. Lewis BS, Swain P. Capsule endoscopy in the evaluation of patients with suspected small intestinal bleeding: Results of a pilot study. Gastrointest Endosc 2002;56:349353.

14. Ell C, Remke S, May A, Helou L, Henrich R, Mayer G. The first prospective controlled trial comparing wireless capsule endoscopy with push enteroscopy in chronic gastrointestinal bleeding. Endoscopy 2002;34:685-689.

15. Mylonaki M, Fritscher-Ravens A, Swain P. Wireless capsule endoscopy: a comparison with push enteroscopy in patients with gastroscopy and colonoscopy negative gastrointestinal bleeding. Gut 2003;52:1122-1126.

16. Waye JD. Small-bowel endoscopy. Endoscopy 2003;35:15-21.

17. Yamamoto H, Yano T, Kita H, Sunada K, Ido K, Sugano K. New system of double-balloon enteroscopy for diagnosis and treatment of small intestinal disorders. Gastroenterology 2003;125:1556; author reply 1556-1557.

18. Suzuki T, Matsushima M, Okita I, Ito H, Gocho S, Tajima H, Tokiwa K, et al. Clinical utility of double-balloon enteroscopy for small intestinal bleeding. Dig Dis Sci 2007;52:1914-1918.

19. Conn M. Tumors of the Small Intestine. In: Gastrointestinal Disease: An Endoscopic Approach by Anthony J. DiMarino, Stanley B. Benjamin. Blackwell Science, 1997; 551-566.

20. Appleyard M, Glukhovsky A, Swain P. Wireless-capsule diagnostic endoscopy for recurrent small-bowel bleeding. N Engl J Med 2001;344:232-233.

21. Rey JF, Gay G, Kruse A, Lambert R. European Society of Gastrointestinal Endoscopy guideline for video capsule endoscopy. Endoscopy 2004;36:656-658.

22. Lewis B, Waye J. Bleeding from the small intestine. In: Sugawa C, Schuman B, Lucas C, eds. Gastrointestinal Bleeding. New York : Igaku-Shoin, 1992: 178-88.

23. Park CH, Kim JO, Choi MG, Kim KJ, Kim YH, Kim YS, Kim TI, et al. Utility of capsule endoscopy for the classification of Crohn's disease: a multicenter study in Korea. Dig Dis Sci 2007;52:1405-1409.

24. Chong AK, Taylor A, Miller A, Hennessy O, Connell W, Desmond P. Capsule endoscopy vs. push enteroscopy and enteroclysis in suspected small-bowel Crohn's disease. Gastrointest Endosc 2005;61:255-261.

25. Herrerias JM, Caunedo A, Rodriguez-Tellez M, Pellicer F, Herrerias JM, Jr. Capsule endoscopy in patients with suspected Crohn's disease and negative endoscopy. Endoscopy 2003;35:564-568. 